\title{
Unsteady Interacting Boundary Layer Method
}

\author{
H. Özdemir* \\ Energy research Center of the Netherlands (ECN), 1755LE Petten, The Netherlands \\ A. van Garrel ${ }^{\dagger}$ \\ University of Twente, 7500AE, Enschede, The Netherlands \\ A. Koodly Ravishankara ${ }^{\ddagger}$ \\ Purdue University, IN 47907, United States \\ H.J. Seubers $₫$ \\ Delft University of Technology, 2629 HT Delft, The Netherlands \\ F. Passalacqua ${ }^{\S}$ \\ Politecnico di Milano, 20133 Milano, Italy
}

Within this study an unsteady, two-dimensional interacting boundary layer method is presented for the incompressible flow around wind turbine rotor blade sections. The main approach is to divide the flow field in to two regions; the one in the vicinity of the surface where the viscosity is effective (so called boundary layer) and the one away from the surface where the flow can be assumed as inviscid. The solutions obtained from these two regions are matched with a quasi-simultaneous viscous-inviscid interaction scheme. For the viscous flow, unsteady integral boundary layer equations together with laminar and turbulent closure sets are solved employing a high-order quadrature-free discontinuous Galerkin method. Laminar to turbulent transition is modeled with the $e^{N}$ method. The potential flow is solved by using the linear-strength vortex panel method. It is shown that introducing the interaction scheme leads to non-conservative mechanisms in the system. The discontinuous Galerkin method is extended to handle these non-conservative flux terms. Furthermore it is shown that this numerical method achieves the designed order of accuracy for smooth problems. Results are presented for the individual numerical solution methods which are verified on various test cases and subsequently for the coupled system which is applied on a chosen test case. Evaluation of a laminar flow over an airfoil section is shown and the results (converged to a steady state solution) are compared with other numerical solutions as well as with the experimental data where available. It is shown that the results of the developed numerical solution method are in good agreement with the experimental data and other computational methods.

Nomenclature $\quad C_{\tau} \quad$ shear stress coefficient

$d \quad$ space dimension

E External flow solution

n unit normal vector

$H^{*} \quad$ shape factor for kinetic energy thickness

solution vector

test functions

$\mathbf{v}$

B

Boundary layer flow solution

$H_{n}$

basis functions

$C_{D} \quad$ dissipation coefficient

$C_{f} \quad$ skin friction coefficient

$C_{\tau_{E Q}} \quad$ shear stress coefficient for equilibrium

I Interaction law

$N \quad$ amplification rate

$N_{w} \quad$ number of wake panels

$R e_{\theta} \quad$ Reynold number based on momentum thickness

\footnotetext{
*Researcher, Wind Energy Unit, Westerduinweg 3, h.ozdemir@ecn.nl. Member AIAA

${ }^{\dagger}$ Researcher, Department of Mechanical Engineering, Engineering Fluid Dynamics, P.O. Box 217, a.vangarrel@utwente.nl

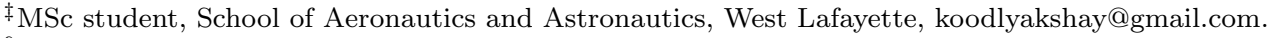

$\S$ MSc student, Aeronautical Engineering, Piazza Leonardo da Vinci, 32, passalacqua.federico@gmail.com.

`MSc student, Aerospace Engineering, Kluyverweg 2, henkseubers@gmail.com.
} 


$\begin{array}{ll}T_{u} & \text { Free-stream turbulence } \\ u_{e} & \text { (boundary layer) edge velocity } \\ u_{p} & \text { x-component of the velocity at a panel } \\ U_{s} & \text { slip velocity } \\ u_{\infty} & \text { free-stream velocity } \\ w_{p} & \text { z-component of the velocity at a panel } \\ \mathrm{H} & \text { shape factor }\end{array}$

Subscripts

$h$ approximation

Symbols

$\delta \quad$ boundary layer thickness

$\delta^{*} \quad$ displacement thickness
$\delta^{k} \quad$ energy thickness
$\gamma \quad$ vorticity strength
$\gamma_{h} \quad$ element boundary
$\lambda$ eigenvalues
$\nu \quad$ kinematic viscosity
$\Omega \quad$ solution (sub) domain
$\Omega_{e} \quad$ element volume
$\sigma_{i} \quad$ source strength
$\theta \quad$ momentum thickness

\section{Introduction}

The Flow around wind turbines are usually unsteady mainly due to natural time dependent changes in the (wind) flow field, i.e. flow separation from the blades or body, high turbulence levels in the flow field or by changes in the position or orientation of the blades, caused by control actuators or by interactions between the fluid and the elastic structure. Important unsteady aspects involve not only the kinematic changes in boundary conditions caused by the motion of a body, but the influence of an unsteady wake, especially in wind farm configuration, when many turbines have to operate relatively close to each other.

If better aerodynamic models are already available in the design phase, significant improvement in terms of performance, control, efficiency and maintenance can be achieved. Apparently, an accurate modeling of the flow around the blades and in the wakes of the wind turbine rotor is required in order to satisfy such requirements.

Current design tools for rotor aerodynamics are mainly based on the Blade Element-Momentum (BEM) approach. These methods, based on local blade section aerodynamics combined with an azimuthal averaged momentum balance, provide quick and efficient estimates. However the range of applicability is relatively small, due to the fundamental assumptions: it is limited to steady state, yaw aligned uniform flow, quasi$2 \mathrm{D}$ blade aerodynamics, spanwise independent induction, without rotor cone angle. To overcome these limits, many empirical corrections have been applied. These include the empirically and theoretically well established tip and root loss corrections, as well as corrections that conflict with the basic assumptions (skewed wake correction, dynamic stall models). Such empirical corrections may improve the model for some situations, but limited predictive confidence can be gained. This especially applies to predicting many relevant phenomena that could influence the design (i.e. load oscillations, dynamic stall, wake interference).

Other approaches, such as inviscid panel methods, ${ }^{1,2}$ nonlinear lifting line methods ${ }^{3}$ and viscous-inviscid splitting methods are based on a less severe set of assumptions and can predict these physical phenomena intrinsically (without corrections). Hence they can be applied to a wider range of rotor operating conditions. The most accurate of these approaches is the splitting of the flow domain into viscous and inviscid regions. Since the introduction of the boundary layer theory by Prandtl in 1904, this approach has been used in many variants, since both regions allow a range of models to be selected, which can be combined using a range of interaction methods (see e.g. ${ }^{4}$ ). Historically, the two regions were solved separately and glued together (weak or no interaction). This approach works for attached flows, but leads to singularities associated with flow separation, both for steady $\left(\right.$ Goldstein ${ }^{5}$ ) and unsteady flows (Van Dommelen and Shen ${ }^{6}$ ). The latter result was interpreted by Matsushita et al. ${ }^{7}$ as the result of discontinuities in the boundary layer (analogous to shocks in compressible flow). They used a dissipative finite difference method to capture separating flow including the singularity.

Later approaches take into account the two-way coupling of the viscous and inviscid regions during computation (strong interaction), thereby successfully extending the validity to (mildly) separated flow. The interaction of the two systems is essentially stabilizing: the breakdown due to singularity does not occur until more strongly separated flow regimes are entered, as can be seen in the analysis of Coenen. ${ }^{8}$ These approaches saw major development in the 1980s, with the quasi-simultaneous ${ }^{9}$ and fully simultaneous ${ }^{10}$ approach. The XFOIL code ${ }^{11}$ is a well known example, for $2 \mathrm{D}$ steady flow around an airfoil. For rotor 
aerodynamics, this code has been extended into RFOIL, ${ }^{12}$ which includes corrections for rotational effects on the boundary layer as introduced by Snel et.al. ${ }^{13}$ This improves the prediction of stall delay, thereby overcoming some limitations of the quasi-2D assumption. Other fully $3 \mathrm{D}$ methods also exist, developed by Nishida, ${ }^{14}$ Coenen $^{8}$ and very recently by Drela. ${ }^{15}$ Few truly unsteady applications are also noted: a dynamic stall simulation by Cebeci ${ }^{16}$ and a flutter computation by Zhang and Liu, ${ }^{17}$ both of which show significant effects due to the viscous part. A more recent method $^{18}$ includes an unsteady formulation which seems very promising, but no validation results have emerged yet.

Unsteady effects are expected to become important near separation since the timescale of the boundary layer increases and the characteristics form envelopes around separated regions and information propagates more slowly. High pitch rates, aeroelastic behavior and wake interference further decrease the timescale at which unsteady effects play a role. Another reason to search for unsteady solutions is that steady solutions may simply not exist for separated flows (e.g. the Von Karman vortex street behind airfoils with a thicker trailing edges.). The nonconservative effects are expected in all (quasi-)simultaneous integral formulations, affecting the unsteady behavior and the location of equilibria. In effect this causes the resulting solutions to depend not only upon the physics of the problem but on the numerical strategy as well. ${ }^{19}$ To prevent this undesirable condition, specialized nonconservative numerical schemes should be applied.

Within this study an unsteady interacting boundary layer method is presented. Unsteady integral boundary layer equations together with laminar and (unsteady) turbulent closure equations are solved by using the discontinuous Galerkin method developed for an arbitrary order of accuracy in space and 4-th order explicit Runge-Kutta scheme in time. Furthermore the numerical method is extended for the non-conservative flux. A linear-strength vortex panel method is employed for the inviscid flow field and both viscous and inviscid solutions are coupled by a quasi-simultaneous interaction scheme introduced by Veldman. ${ }^{9}$

In the next section the details of the method and the numerical solutions are presented. In section (III) the numerical results of various verification and validation test cases is shown and finally conclusions are summarized in section (IV).

\section{Unsteady interacting boundary layer method}

For large varieties of flow conditions, the Navier-Stokes equations together with turbulence models deliver accurate solutions both for steady and unsteady cases. The main issue arises when large scale differences exist in the flow field and the time dependency can not be avoided where solving the Navier-Stokes equations become computationally too expensive. Especially when the design phase of wind turbine blades are considered, these computational times become critical and makes the use of full flow-field solutions impractical.

When the flow considered is at a very high Reynolds number or with a very small viscosity we have the flexibility of making simplications to the Navier-Stokes equations. Prandtl showed in 1904 how viscosity effects the Reynolds number and for this limiting case he derived the boundary layer equations. However this reduced form of equations still represent the flow field variables in the number of space dimension under consideration (see i.e. ${ }^{20}$ ). When the special case of designing airfoil sections of a blade considered in order to predict the loading on these (wind turbine) blades we do not need to resolve the flow field in detail but rather we are interested in certain integral quantities of the boundary layer and their effects on the pressure distribution. These integral values depend on the lengths $x$ and $y$ along the surface in three-dimensions and only the length $x$ in two-dimensions. For the global description of the boundary layer these integral values are obtained by integrating the boundary layer equations with respect to the direction normal to the no-slip surface, $z$, over the boundary layer thickness that leads to the integral boundary layer equations.

Integral boundary layer equations have been used widely for the global description of the flow $21,22,23$ especially in engineering applications for aircraft aerodynamics. The methods based on these equations have the advantage of lowering the space dimension by one, and as a consequence there is no need for a volume grid. This leads to a reduction in computational costs and input efforts. At the start of the CFD era these methods therefore were used as airfoil analysis tools and even today some successful applications are based on this approach. ${ }^{11}$ Integral boundary layer equations have been commonly discretized with Finite-Difference schemes. ${ }^{24,10}$ More recently also Finite-Element and Finite-Volume discretizations are employed. ${ }^{25}$

In the remainder of this section, individual components that form the unsteady interacting boundary layer are presented in details. The unsteady form of integral boundary layer equations and the linear vorticity panel method are presented that are the solution for the viscous and inviscid flow regions respectively. Furthermore, the interaction scheme is briefly explained and the complete set of equations are summarized. 
Finally the numerical solution of the equation set is given.

\section{II.A. Integral boundary layer method}

The integral boundary layer equations (IBL) can be derived starting from the unsteady, two-dimensional boundary layer equations with a fundamental assumption that the only effect of the boundary layer and the wake is to displace the inviscid flow away from the physical body to create an effective displacement thickness. Lighthill ${ }^{26}$ showed that this assumption is accurate when the ratio of the boundary layer thickness to streamline radius of curvature is small. This assumption is valid for most aerodynamic flows of interest. At the trailing edge region there is a possibility where the accuracy of this assumption is arguable. The streamline curvature at the trailing edge or impingement of a shock wave can cause a significant normal pressure gradient in the boundary layer where whole boundary layer approximation breaks down. To obtain these useful integral values for the global description of the boundary layer the boundary layer equations should be integrated with respect to the transverse direction $\mathrm{z}$ (normal to the no-slip surface) over the boundary layer thickness. The integral boundary layer equations can be derived either starting from a control volume and setting up the mass and momentum balance or from the boundary layer equations and integrating over the boundary layer thickness.

In the literature the derivation of the integral boundary layer equations are given in various ways where one is slightly differing from another (i.e. see references ${ }^{27,28,29}$ ). It is interesting to note that in a recent study, Seubers ${ }^{30}$ showed the derivation of the integral boundary layer equations with a streamline based control volume approach including terms for non-classical (separating) boundary layers.

Integrating the following equation for $n=0,1, \ldots$ with respect to the transverse direction (normal to the no-slip surface) over the boundary layer thickness, we can derive the $n$-th moment of boundary layer equation:

$$
\text { momentum equation } \times(n+1) u^{n}-\text { continuity equation } \times\left(u_{e}^{n+1}-u^{n+1}\right),
$$

in which the free-stream velocity $u_{e}=u_{e}(x, t)$ is presumably known from a potential-flow analysis.

In the current study the 0-th (momentum integral) and 1-st (kinetic-energy integral) moments of momentum equations are used to obtain the global quantities such as displacement, momentum and energy thicknesses of the boundary layer as given below

$$
\begin{aligned}
\frac{\partial \delta^{*}}{\partial t}+\frac{\partial}{\partial x}\left(u_{e} \theta\right) & =\frac{C_{f}}{2} u_{e}-\left(\delta^{*}+\theta\right) \frac{\partial u_{e}}{\partial x}-\frac{\delta^{*}}{u_{e}} \frac{\partial u_{e}}{\partial t}, \\
\frac{\partial\left(\delta^{*}+\theta\right)}{\partial t}+\frac{\partial}{\partial x}\left(u_{e} \delta^{k}\right) & =C_{D} u_{e} 2 \delta^{k} \frac{\partial u_{e}}{\partial x}-2 \frac{\theta}{u_{e}} \frac{\partial u_{e}}{\partial t},
\end{aligned}
$$

where $\delta^{*}$ is the displacement thickness, $\theta$ is the momentum thickness, $\delta^{k}$ is the energy thickness, $C_{f}$ is the friction coefficient, $C_{D}$ is the viscous diffusion coefficient and $u_{e}$ is the velocity at the edge of the boundary layer.

The system of Eqs. (1) and (2) are accurate for laminar flows and for turbulent flows when the turbulence production and dissipation are in near equilibrium. In such a scenario, the dissipation coefficient, $C_{D}$ depends only on local boundary layer parameters. However, experiments (eg Goldberg ${ }^{31}$ ) show that there are significant upstream history effects on Reynolds stresses for flows with adverse pressure gradients. These effects are included by introducing Reynolds stress an additional unknown in a stress-transport equation as described by Green's lag-entrainment method. ${ }^{32}$ Özdemir $^{33}$ derived the unsteady form of this so called shear-lag equation and in a recent study it is further simplified by $\mathrm{Ye}^{34}$ to take the following form:

$$
\frac{\partial\left(\frac{u_{e}}{u} C_{\tau}\right)}{\partial t}+\frac{\partial\left(u_{e} C_{\tau}\right)}{\partial x}=\frac{C_{\tau} u_{e}}{\delta} K_{c}\left(C_{\tau_{E Q}}^{\frac{1}{2}}-C_{\tau}^{\frac{1}{2}}\right)-\frac{u_{e}}{u} \frac{2 C_{\tau}}{u_{e}} \frac{\partial u_{e}}{\partial t}-C_{\tau} \frac{\partial u_{e}}{\partial x},
$$

with $C_{\tau}$ the shear stress coefficient, $C_{\tau_{E Q}}$ shear stress coefficient for the equilibrium flow conditions and

$$
K_{c}=2 a_{1} \frac{u_{e}}{u} \frac{\delta}{L}
$$

and

$$
\delta=\theta\left(3.15+\frac{1.72}{H-1}\right)+\delta^{*}
$$


The commonly used values are $\frac{u_{e}}{u}=1.5$ and $\frac{\delta}{L}=12.5$ but Thomas ${ }^{35}$ takes in to account the dependency on the shape factor and gives $\frac{u_{e}}{u}=\frac{3 H}{H+2}$, which is more accurate for separated flow profiles.

Vector form of Eqs. (1), (2) and (3) can be formulated as:

$$
\frac{\partial \mathbf{u}}{\partial t}+\frac{\partial}{\partial x}[\mathbf{f}(\mathbf{u})]=\mathbf{s}(\mathbf{u}),
$$

where the new conservation variable $\mathbf{u}$, flux $\mathbf{f}(\mathbf{u})$ and source $\mathbf{s}(\mathbf{u})$ have been introduced:

$$
\mathbf{u}=\left[\begin{array}{c}
\delta^{*} \\
\delta^{*}+\theta \\
\frac{u_{e}}{u} C_{\tau}
\end{array}\right], \quad \mathbf{f}(\mathbf{u})=\left[\begin{array}{c}
u_{e} \theta \\
u_{e} \delta^{k} \\
u_{e} C_{\tau}
\end{array}\right], \quad \mathbf{s}(\mathbf{u})=\left[\begin{array}{c}
\frac{C_{f}}{2} u_{e} \delta^{*}+\theta \frac{\partial u_{e}}{\partial x}-\frac{\delta^{*}}{u_{e}} \frac{\partial u_{e}}{\partial t} \\
C_{D} u_{e}-2 \delta^{k} \frac{\partial u_{e}}{\partial x}-2 \frac{\theta}{u_{e}} \frac{\partial u_{e}}{\partial t} \\
\frac{C_{\tau} u_{e}}{\delta} K_{c}\left(C_{\tau_{E Q}}^{\frac{1}{2}}-C_{\tau}^{\frac{1}{2}}\right)-\frac{u_{e}}{u} \frac{2 C_{\tau}}{u_{e}} \frac{\partial u_{e}}{\partial t}-C_{\tau} \frac{\partial u_{e}}{\partial x}
\end{array}\right] .
$$

The above equation set forms a conservation form when the interaction scheme is not considered and the system is shown to be hyperbolic. ${ }^{36,20}$

\section{II.A.1. Laminar to Turbulent transition}

Within this study laminar to turbulent transition is modeled by the $e^{N}$ method. The $e^{N}$ (originally $e^{9}$ ) method was introduced by van Ingen ${ }^{37}$ and Smith. ${ }^{38}$ Based on the linear stability theory, ${ }^{34}$ this semiempirical method provides a good practical prediction of natural transition onset for incompressible twodimensional boundary layers.

The prediction of the natural transition is based on the evolution of small disturbances introduced in the laminar flow. An exponential decay of disturbances with time, implies the mean flow remains laminar and is stable. However, if the disturbances increase with time, the flow is considered unstable and transitions to turbulent flow. ${ }^{39}$

The $N$ in the $e^{N}$ method stands for the maximum amplification rate of the disturbances at a given location. The flow is said to transition to a turbulent regime, at that location, when this amplification rate $(N)$ exceeds a critical value $\left(N_{\text {crit }}\right)$ determined from experiments. In this study, the value of this critical amplification rate is adopted from Drela: ${ }^{40}$

$$
N_{\text {crit }}=-8.43-2.4 \ln \frac{T u}{100} .
$$

$T u$ is the free-stream turbulence intensity nominally taken as $0.07 \%$ for airfoils in wind tunnel conditions. The amplification rate $N$ at any location is determined by:

$$
N=\frac{d N}{d R e_{\theta}}\left(R e_{\theta}-R e_{\theta_{c} r i t}\right)
$$

where $R e_{\theta}$ is the Reynolds number based on momentum thickness, $\theta$,

$$
R e_{\theta}=\frac{\theta u_{e}}{\nu} .
$$

The slope is given by Drela ${ }^{10}$

$$
\frac{d N}{d \operatorname{Re}_{\theta}}=0.028(H-1)-0.0345 e^{-\left(3.87 \frac{1}{H-1}-2.52\right)^{2}} .
$$

$\mathrm{Ye}^{34}$ slightly modified the $R e_{\theta_{\text {crit }}}$ that is based on Arnal's experimental work ${ }^{41}$ to find the onset as follows

$$
\log \left(\operatorname{Re}_{\theta_{\text {crit }}}\right)=\left(\frac{0.267659}{H-1}+0.394429\right) \tanh \left(\frac{12.7886}{H-1}-8.57463\right)+\frac{3.04212}{H-1}+0.6660931 .
$$

For similar flows, $H$ is constant and $R_{e}$ is uniquely related to the stream-wise coordinate $x$. Eq. (8) immediately gives the amplification factor $\mathrm{N}$ as a unique function of $x$. 
For the unsteady form of the IBL equations amplification rate can be modified to contain the time dependent term:

$$
\Delta N=\frac{\partial N}{\partial t} \Delta t+\frac{\partial N}{\partial x} \Delta x
$$

to give

$$
\Delta N=\frac{\partial N}{\partial R e}\left(\frac{\partial R e_{\theta}}{\partial t} \Delta t+\frac{\partial R e_{\theta}}{\partial x} \Delta x\right) .
$$

For the solution of the IBL equations the last line of Eq. (7) is replaced by the Eq. (14) for the laminar region of the flow field to check the transition to the turbulent flow. The complete system can be written in vector form for the laminar system can be written as follows:

$$
\mathbf{u}=\left[\begin{array}{c}
\delta^{*} \\
\delta^{*}+\theta \\
0
\end{array}\right], \quad \mathbf{f}(\mathbf{u})=\left[\begin{array}{c}
u_{e} \theta \\
u_{e} \delta^{k} \\
N
\end{array}\right], \quad \mathbf{s}(\mathbf{u})=\left[\begin{array}{c}
\frac{C_{f}}{2} u_{e} \delta^{*}+\theta \frac{\partial u_{e}}{\partial x}-\frac{\delta^{*}}{u_{e}} \frac{\partial u_{e}}{\partial t} \\
C_{D} u_{e}-2 \delta^{k} \frac{\partial u_{e}}{\partial x}-2 \frac{\theta}{u_{e}} \frac{\partial u_{e}}{\partial t} \\
\frac{\partial N}{\partial e}\left(\frac{\partial R e_{\theta}}{\partial t} \Delta t+\frac{\partial R e_{\theta}}{\partial x} \Delta x\right)
\end{array}\right] .
$$

When $N$ value is equal to the defined $N$ crit value, the onset of transition is triggered and the equations will switch to the turbulent ones (Eq. (7)). Alternatively the equation for $N$ can be applied as a check after each time step.

\section{II.A.2. Closure set}

The integration over the direction normal to the surface applied in order to derive the IBL equations removes some physical information. In the equation set (15) the displacement thickness $\delta^{*}$, kinetic energy thickness $\delta^{k}$, momentum thickness $\theta$, friction coefficient $C_{f}$, viscous diffusion coefficient $C_{D}$ and the edge velocity $u_{e}$ are unknowns. Assuming that the edge velocity is provided by the potential flow solver there are still 3 more unknowns than the number of equations. In order to close this system the so-called closure relations are introduced. These closure relations can be derived by modeling unknowns in terms of other unknowns using experimental data or analytical solutions of representative test cases under certain assumptions (e.g. Drela, ${ }^{10}$ Nishida $\left.^{14}\right)$.

\section{Laminar closure set}

Within this study the laminar closure relations that are given by Nishida ${ }^{14}$ and Drela ${ }^{10}$ are used for the computation of laminar boundary layer flows. The shape factor, $H$ for the displacement thickness and the shape factor, $H^{*}$ for the kinetic energy thickness are defined as follows

$$
H=\frac{\delta^{*}}{\theta}, \quad H^{*}=\frac{\delta^{k}}{\theta} .
$$

Althought the closure set for laminar system can be found from the literature for the sake of completeness they are given here as well:

$$
\begin{gathered}
H^{*}= \begin{cases}0.0111 \frac{(H-4.35)^{2}}{H+1.0}-0.0278 \frac{(H-4.35)^{3}}{H+1.0}+1.528-0.0002[(H-4.35)]^{2} & H<4.35, \\
0.015 \frac{(H-4.35)^{2}}{H+1.0}+1.528 & H \geq 4.35,\end{cases} \\
\operatorname{Re}_{\theta} C_{f}= \begin{cases}0.0727 \frac{(5.5-H)^{3}}{H+1.0}-0.07 & H<5.5, \\
0.015\left(1.0-\frac{1}{H-4.5}\right)^{2}-0.07 & H \geq 5.5,\end{cases} \\
\frac{\operatorname{Re}_{\theta} C_{D}}{H^{*}}= \begin{cases}0.207+0.00205(4.0-H)^{5.5} & H<4.0, \\
0.207+0.0016 \frac{(H-4.0)^{2}}{1.0+0.02(H-4.0)^{2}}(4.0-H)^{5.5} & H \geq 4.0 .\end{cases}
\end{gathered}
$$


The accuracy of the IBL system is bounded by the accuracy of the closure relations. The analytical models used for the closure relations do not include unsteady effects and experimental data are correlated with trial and error curve-fits, which might bring non-negligible fit-errors. Due to the complex form of the closure relations, the high order numerical method applied will also have limited order of accuracy, as it will be discussed later.

The laminar system is analyzed in details previously $\left(\sec ^{36}\right.$ and $\left.^{20}\right)$ and it is shown that both eigenvalues are positive for low values of $H$ until a point of separation, where $\partial H^{*} / \partial H=0$ and $H=4.19808$. Downstream of this point the flow is separated and one of the characteristics travels in the upwind direction. As noted first by Goldstein in $1948,{ }^{5}$ at the point of separation solution is singular. This should not constitute a problem for the unsteady application since inversion of the operating matrix is avoided. However, as discussed by van Dommelen and Shen the unsteady method presented is not able to reach the correct steady solution for separating flows, due to the spontaneous generation of the singularity in a separating laminar boundary layer. ${ }^{6}$ One way to avoid this problem is to couple the viscous method analyzed here with an inviscid one which brings the necessity of a viscous-inviscid interaction (VII) scheme where the interaction changes the equations to be solved but allows the computation of the correct solution also for separated flows.

\section{Turbulent closure set}

Turbulent boundary layers have a two-layer structure (and an overlap region), the thickness of which scales differently with the local Reynolds number $R e_{\theta}$, a one-parameter velocity profile is not adequate to describe all turbulent boundary layers and such a dependency on $R e_{\theta}$ must be considered. Many closure sets can be found in literature for the turbulent closure set as well. For the present study the closure sets from Drela and Giles ${ }^{42}$ and Nishida ${ }^{14}$ are taken, with small corrections suggested by $\mathrm{Ye}^{34}$ and neglecting compressibility effects.

The skin friction coefficient $C_{f}$ is given by:

$$
C_{f}=0.3 e^{-1.33 H}\left(\frac{\log R e_{\theta}}{2.3026}\right)^{-1.74-0.31 H}+0.00011\left[\tanh \left(4.0-\frac{H}{0.875}\right)-1.0\right] .
$$

For the energy thickness shape factor $H^{*}$ the following relation is used:

$$
H^{*}= \begin{cases}1.505+\frac{4}{R e_{\theta}}+\left(0.5-\frac{4}{R e_{\theta}}\right)\left(\frac{H_{0}-H}{H_{0}-1.0}\right)^{2} \frac{1.5}{H+0.5}, & H<H_{0}, \\ 1.505+\frac{4}{R e_{\theta}}+\left(H-H_{0}\right)^{2}\left[0.007 \frac{\log R e_{\theta}}{\left.H-H_{0}+\frac{4}{R e_{\theta}}+\frac{0.015}{H}\right],} \quad H \geq H_{0},\right.\end{cases}
$$

where $H_{0}$ is defined as

$$
H_{0}=\min \left(3+\frac{400}{R e_{\theta}}, 4\right) .
$$

Equilibrium shear stress coefficient

$$
C_{\tau_{E Q}}=\frac{H^{*}}{2} \frac{0.03}{1-U_{s}}\left(\frac{H-1}{H}\right)^{3} .
$$

Dissipation coefficient

$$
C_{D}=\frac{C_{f}}{2} U_{s}+C_{\tau}\left(1-U_{s}\right) .
$$

with the slip velocity

$$
U_{s}=\frac{H^{*}}{6}\left(\frac{4}{H}-1\right),
$$




\section{II.B. Linear-strength vortex panel method}

For the solution of the inviscid region a panel method based on constant strength doublets and sources can be used. But as argued by Katz and Plotkin ${ }^{43}$ and also as stated in the previous studies ${ }^{20,44}$ use of constant strength doublets and sources leads a slight disagreement near the maximum suction area and near the rear stagnation point. Katz and Plotking suggests to use either a grid stretching towards the trailing edge or to use the velocity formulation for the Kutta condition. Within this study we employed linear-strength vortex panel method instead to overcome this problem which is also a higher order panel method. The panel surface is assumed to be planar and the singularity strength will change linearly along the panel (see Fig. (1)). Consequently, the singularity strength on each panel includes two unknowns and additional equations need to

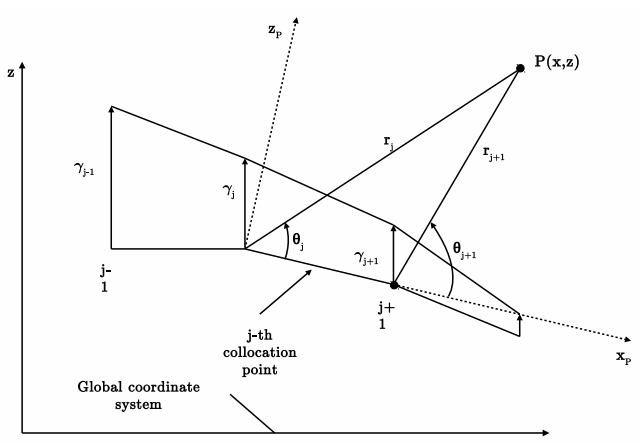

Figure 1. Nomenclature for linear strength singularity panel. ${ }^{43}$ be formulated. The detailed derivations can be found in the work of Koodly Ravishankara ${ }^{44}$ ans is not repeated here.

The velocity components at a location $P(x, z)$ due to a panel $j$, with vorticity strengths varying from $\gamma_{j}$ to $\gamma_{j+1}$ in panel coordinates is expressed as: ${ }^{43}$

$$
\begin{aligned}
u_{p}= & \frac{z}{2 \pi}\left(\frac{\gamma_{j+1}-\gamma_{j}}{x_{j+1}-x_{j}}\right) \ln \frac{r_{j+1}}{r_{j}} \\
& +\frac{\gamma_{j}\left(x_{j+1}-x_{j}\right)+\left(\gamma_{j+1}-\gamma_{j}\right)\left(x-x_{j}\right)}{2 \pi\left(x_{j+1}-x_{j}\right)}\left(\theta_{j+1}-\theta_{j}\right), \\
w_{p}= & \frac{z}{2 \pi}\left(\frac{\gamma_{j+1}-\gamma_{j}}{x_{j+1}-x_{j}}\right)\left[\frac{x_{j+1}-x_{j}}{z}+\left(\theta_{j+1}-\theta_{j}\right)\right] \\
& -\frac{\gamma_{j}\left(x_{j+1}-x_{j}\right)+\left(\gamma_{j+1}-\gamma_{j}\right)\left(x-x_{j}\right)}{2 \pi\left(x_{j+1}-x_{j}\right)} \ln \frac{r_{j}}{r_{j+1}} .
\end{aligned}
$$

The Eqs. (26) can be used to compute the influence coefficients for all the panels. Thus we have $N+1$ unknown singularity strengths for $N$ panels. A zero normal velocity condition is enforced to form the required equations. Denoting the influence on $i-t h$ panel by the $j-t h$ panel with vorticity strengths $\gamma_{j}$ to $\gamma_{j+1}$ as $a_{i j}$ and $a_{i j+1}$, we can write the condition for zero normal velocity at the first panel as

$$
a_{11} \gamma_{1}+a_{12} \gamma_{2}+\ldots+a_{1 N+1} \gamma_{N+1}=u_{\infty} \sin (\alpha \theta)
$$

where $u_{\infty}$ is free stream velocity, $\alpha$ is the angle of attack and $\theta$ is the panel angle.

A similar equation can be written for all $N$ panels, and the Kutta condition (Eq. 28) provides the $N+1^{\text {th }}$ equation.

$$
\gamma_{1}+\gamma_{N+1}=0 .
$$

The system of $N+1$ equations can be solved for the unknown vorticity strengths $\gamma_{1}$ to $\gamma_{N+1}$. The edge velocity distribution is then found by using the influence coefficients for tangential velocity from Eqs. (26) along with the contribution of free stream velocity, ${ }^{43}$

$$
u_{e_{i}}=\sum_{1}^{N+1} a_{i j} \gamma_{j}+u_{\infty} \cos (\alpha-\theta) .
$$

\section{Computation of wake geometry}

The wake geometry can be determined once the inviscid solution is found. The first point on the wake is taken to be a very small distance from the trailing edge and subsequent points are found using the constraint that there is no flow across the wake. First, the velocity at the first trailing edge point is determined and subsequent points can be found by moving in the direction of the velocity at previous points. 


\section{Effect of boundary layer}

To incorporate the effect of boundary layer in the potential flow method the concept of transpiration velocity is used. The viscosity which is effective only in the region around the airfoil, reduces the velocity in the vicinity. Since there must be a constant volume flow per unit span between the surface and the streamline just outside the boundary layer, due to continuity requirements, the flow reduction inside the boundary layer must be compensated for by an outward displacement of such a streamline of a distance $\delta^{*}$, whose value at any point of the surface can be calculated, to a first approximation, directly from the integral boundary layer equations, this effect should be taken into account. The additional outflow ${ }^{8}$ is as if the flow around the body were supplemented by the effect of a surface distribution of sources, whose strength per unit area is:

$$
\sigma_{i}=\frac{d}{d x}\left(u_{e} \delta^{*}\right), \quad i=1,2 . . N+N_{w}
$$

where $N$ is the number of panels on the airfoil and $N_{w}$ is the number of panels on the wake. The new edge velocity distribution is then found ${ }^{11}$ as

$$
u_{e_{i}}=u_{I N V_{i}}+\sum_{1}^{N+N_{w}} b_{i j} \sigma_{j} .
$$

$u_{I N V_{i}}$ is the value from Eq. (29) and $b_{i j}$ are the influence coefficients of sources and $\sigma_{j}$ is given in Eq. (30).

\section{II.C. Viscous-inviscid interaction scheme}

The equations of motion in each of the subdomain provide a relation between the velocity along the edge of the boundary layer $u_{e}$ and the displacement thickness $\delta^{*}$. A symbolic notation is here used to denote these relations:

$$
\begin{array}{ll}
\text { external flow: } & u_{e}=E\left[\delta^{*}\right], \\
\text { boundary-layer flow: } & u_{e}=B\left[\delta^{*}\right],
\end{array}
$$

where $E$ denotes "external" and $B$ "boundary layer". The inverse form of the boundary layer formulation has been indicated with $B$ since it is assumed that this formulation does possess a (unique) solution after discretisation even at separation points, while the direct one does not. ${ }^{46}$ If a steady solution is sought, the two edge velocities for given displacement thickness need to match. A justification of this requirement is also given by the functional approach theory for steady flows, extensively treated by Brune et al. ${ }^{47}$ and Williams and Smith. ${ }^{48}$ In order to decrease the computational effort required by the simultaneous method but preserving the asset to allow a strong interaction between the layers, based on the triple-deck theory, the quasi-simultaneous method was developed by Veldman. ${ }^{9}$ The idea behind this scheme is to combine the advantages of the direct and simultaneous methods, and avoiding the singularity problems at separation. Instead of solving viscous and inviscid regions simultaneously, the boundary layer calculations are performed together with an approximation for the external

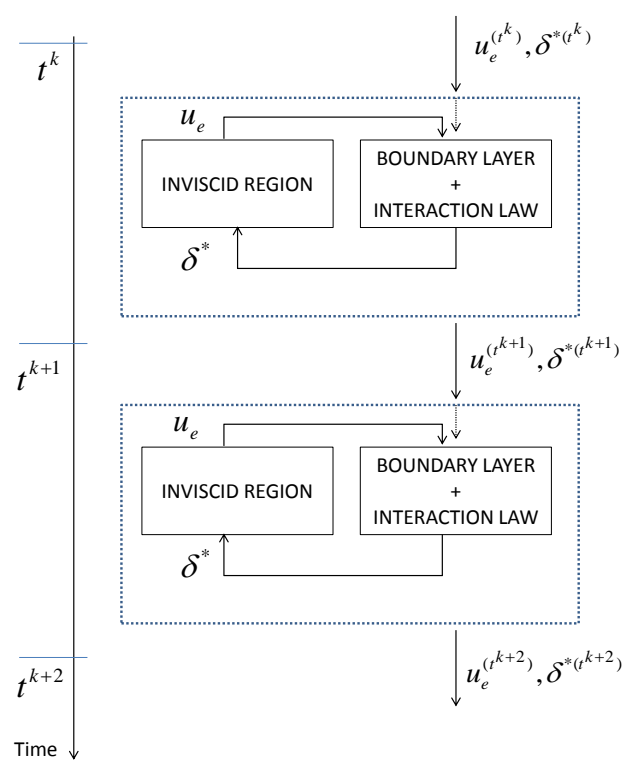
inviscid flow. The boundary layer is therefore informed instantaneously about how the external flow reacts on changes inside the boundary layer itself through a simple, but sufficiently accurate approximation of the external flow, called interaction law, and denoted here with $I$. The following iterative process is therefore created:

$$
\left\{\begin{array}{l}
\left.u_{e}^{(n+1)}-I\left[\delta^{*(n+1}\right)\right]=E\left[\delta^{*(n)}\right]-I\left[\delta^{*(n)}\right], \\
u_{e}^{(n+1)}-B\left[\delta^{*(n+1)}\right]=0
\end{array}\right.
$$


Since the goal of the interaction law is to make the numerical calculations survive the separation point, a good description of the local interactive physics is essential. When this requirement is fulfilled, a fast convergence can be pursued. It is remarked by Veldman that the choice of the interaction law does not influence the converged solution. This is in fact only determined by the choices made for the inviscid and viscous flow operators $E$ and $B$ in Eqs. (32) and (33). The interaction law only controls the rate with which the viscous-inviscid solution is obtained. ${ }^{49}$ In the quasi-simultaneous method the boundary layer is solved together with an approximation of the inviscid flow. Every time-step, iterations are performed in order to reach a converged solution between the edge velocity provided by the boundary layer modelling together with the interaction law and the complete inviscid modelling described by Eq. (34). The stopping criterion can be either based on the difference between the two edge velocities or on a fixed number of iterations. Cebeci ${ }^{16}$ assumed that 20 viscous-inviscid iterations even for flow involving stall are sufficient, while less iterations are required when stall does not occur. Unless an unsteady panel method based on Helmholtz theorem of conservation of vorticity is developed, ${ }^{50}$ a fixed number of iterations is performed. The time advance is kept fixed during the iteration process by fixing the initial conditions to the solution at the previous time-step. Only when the iteration procedure is terminated the solution computed is used as the new initial condition for the next iterations. A schematic of the unsteady quasi-simultaneous method is shown in Fig. ( 2).

Veldman ${ }^{49}$ suggests that a simple thin-airfoil approximation of the external inviscid flow is sufficiently useful for the development of the method. Other choices are possible. The interaction law implemented has the form:

$$
u_{e}(x)=u_{e_{0}}(x)+\frac{1}{\pi} \int_{\chi} \frac{\mathrm{d}}{\mathrm{d} \zeta}\left(u_{e} \delta^{*}\right) \frac{\mathrm{d} \zeta}{x-\zeta}
$$

where $u_{e_{0}}$ is the edge velocity distribution without displacement effects and $\chi$ is the region where strong interaction is required, as indicated by triple-deck theory.

When the edge velocity distribution $u_{e}$ is considered as an additional unknown and the interaction law Eq. (35) is added to the set of Eqs. (7) (or to the laminar version of the mentioned equation), the nature of the problem changes. This can be shown just looking at the integral boundary-layer equations, independent of the interaction law chosen. The terms where the derivatives of $u_{e}$ appear can not be considered sources any more, and by indicating the unknown vector as $\mathbf{u}=\left(u_{1}, u_{2}, u_{3}\right)^{T}=\left(\delta^{*}, \delta^{*}+\theta, u_{e}\right)^{T}$, the integral boundary layer equations are written in the form:

$$
\begin{gathered}
\frac{\partial u_{1}}{\partial t}+\frac{u_{1}}{u_{3}} \frac{\partial u_{3}}{\partial t}+u_{2} \frac{\partial u_{3}}{\partial x}+\frac{\partial}{\partial x}\left[u_{3}\left(u_{2}-u_{1}\right)\right]=\frac{C_{f}}{2} u_{3}, \\
\frac{\partial u_{2}}{\partial t}+\frac{2\left(u_{2}-u_{1}\right)}{u_{3}} \frac{\partial u_{3}}{\partial t}+2 H^{*}\left(u_{2}-u_{1}\right) \frac{\partial u_{3}}{\partial x}+\frac{\partial}{\partial x}\left[u_{3} H^{*}\left(u_{2}-u_{1}\right)\right]=C_{D} u_{3} .
\end{gathered}
$$

Already the above equations present the problem that they contain non-conservative products that cannot be transformed into divergence form, i.e. the equations in the form $\partial_{t} u+\partial_{x} f(u)+g(u) \partial_{x} u=s(u)$ cannot be written as $\partial_{t} u+\partial_{x} h(u)=s(u)$. This causes problems once the solution becomes discontinuous, because the weak solution in the classical sense of distributions then does not exist.

Therefore in order to couple the system described by the integral boundary layer equations with the interaction law, a numerical method for non-conservative systems is required. In the section below the discontinuous Galerkin method is presented including a non-conservative flux scheme for genuinely nonconservative problems.

\section{II.D. Numerical solution}

As discussed above introducing the boundary layer edge velocity $u_{e}$ as an unknown to the integral boundary layer equations (either to laminar or turbulent set) by means of the (quasi-)simultaneous viscous-inviscid interaction scheme leads to a non-conservative system of equations independent of the chosen interaction scheme. This leads to a need of a special treatment of the chosen numerical solution method namely it is required to introduce a non-conservative flux scheme. Within this study the IBL equations together with the closure set are solved with a high-order discontinuous Galerkin (DG) method. In various literature ${ }^{51,52}$ as well as in the previous studies ${ }^{53,36,54,20}$ the conservative form of discontinuous Galerkin method is presented in details for conservative systems. Within this study the DG method applied to non-conservative hyperbolic systems is briefly discussed.

It has been shown ${ }^{19}$ that the interacting boundary layer equations do not form a set of conservation laws, but rather a more general non-conservative hyperbolic system. The difficulties in defining and modeling 
these type of systems originate from their dependence on the limit of the physical process from which they are derived; in contrast with conservation laws, whose definition and modeling do not require such additional information. In case of the interacting boundary layer, this information is contained in the averaged NavierStokes formulation for the shear layer. The second order diffusive terms, neglected in the hyperbolic system, can be used to determine the correct behavior. This additional physical information affecting the solution is to be implemented in a numerical approximation, more specifically in a discontinuous Galerkin framework. The limiting behavior of the numerical approximation is determined by two aspects: the choice of physical paths in the solution space $\Omega$ (as introduced by Dal Maso, LeFloch and Murat ${ }^{55}$ ), and the choice of numerical test function paths in $\Omega^{*}$ (as introduced by Seubers ${ }^{19}$ ). Existing numerical approaches can be divided into two categories, based on convergence to physically relevant solutions. The well-balanced schemes focus on the use of correct solution paths where available. These schemes preserve equilibrium solutions. The entropy conserving schemes impose an additional conservation principle by construction of a special artificial diffusion term. The existence of the relation between the artificial diffusion terms and the test function paths is a requirement for well-balancing, which is attained when using the physically correct solution paths. Since the exact paths for the interacting boundary layer system are not available analytically, simplified linear solution paths can be used to obtain convergence to the physical solution, as long as the numerical stabilization is consistent with the higher order diffusive terms in the limiting physical process, as in the entropy-stable-path-consistent scheme.

A general hyperbolic non-conservative system can formally be written in the following form:

$$
\frac{\partial \mathbf{u}}{\partial t}+\sum_{i=1}^{d} A_{i}(\mathbf{u}) \frac{\partial \mathbf{u}}{\partial x_{i}}=\mathbf{s}(\mathbf{u}), \quad \text { in }(0, T) \times \Omega,
$$

where $\Omega$ is a bounded sub domain of $\mathbb{R}^{d}, d=1,2$ or $3, \mathbf{u}=\left(u_{1}, u_{2}, \ldots, u_{m}\right)^{T}, \mathbf{s}(\mathbf{u})$ is a source term and the matrices $A_{i}(\mathbf{u}), i=1, \ldots, d$ are such that any linear combination $\sum_{i=1}^{d} \xi_{i} A_{i}(\mathbf{u})$ has $m$ real eigenvalues and a complete set of eigenvectors. The latter property ensures that the system is hyperbolic. ${ }^{56}$ Within the classical DG method based on the conservative expression the flux expressions on element boundaries is provided by means of integration by parts. However, for equations in non-conservative form or if a conservative form does not exist, as it is the case for the system of equations investigated, it is not clear how to perform such integration. Another approach, which describes the DG method as a Galerkin method with element-wise weakly imposed boundary conditions is shown by Hulsen ${ }^{57}$ but still requires integration by parts. The procedure followed here for non-conservative systems is based on the method suggested by Hulsen but instead of integration by parts, introduces extra terms accounting for the jumps across element boundaries. Multiplying Eq. (37) by a test function $\mathbf{v}(\mathbf{x})$ and integrating over the domain $\Omega$ gives:

$$
\int_{\Omega} \mathbf{v}^{T}\left(\frac{\partial \mathbf{u}}{\partial t}+\sum_{i=1}^{d} A_{i}(\mathbf{u}) \frac{\partial \mathbf{u}}{\partial x_{i}}-\mathbf{s}(\mathbf{u})\right) \mathrm{d} \Omega=0, \quad \forall \mathbf{v} \in V,
$$

where $V$ is a a suitable function space for both $\mathbf{u}$ and $\mathbf{v}$, e.g. $\left(H^{1}(\Omega)\right)^{m}$. The space $V$ is then approximated by $V_{h}^{(e)}$. Inserting the approximation in Eq. (38) requires the integral to be split into a sum over the element integrals and a sum across the boundary integrals. The latter terms appear because on the element boundaries the normal component of the derivative of $\mathbf{u}_{h}$ is infinite. The following form is obtained:

$$
\sum_{e=1}^{n_{e}}\left(\int_{\Omega_{e}} \mathbf{v}_{h}^{T}\left(\frac{\partial \mathbf{u}_{h}}{\partial t}+\sum_{i=1}^{d} A_{i}\left(\mathbf{u}_{h}\right) \frac{\partial \mathbf{u}_{h}}{\partial x_{i}}-\mathbf{s}\left(\mathbf{u}_{h}\right)\right) \mathrm{d} \Omega\right)+\int_{\gamma_{h}} \mathbf{v}_{h}^{T} \Delta_{h} \mathrm{~d} \gamma=0,
$$

where $\gamma_{h}=\cup_{e=1}^{n_{e}} \partial \Omega_{e}$, which consists of all element boundaries. The expression of $\Delta_{n}$ is:

$$
\begin{array}{r}
\Delta_{n}=\int_{n^{-}}^{n^{+}} \sum_{i=1}^{d} A_{i}\left(\mathbf{u}_{h}\right) \frac{\partial \mathbf{u}_{h}}{\partial x_{i}} \mathrm{~d} n=\int_{n^{-}}^{n^{+}} \sum_{i=1}^{d} A_{i}\left(\mathbf{u}_{h}\right) n_{i} \frac{\partial \mathbf{u}_{h}}{\partial n} \mathrm{~d} n \\
=\int_{u^{-}}^{u^{+}} \sum_{i=1}^{d} n_{i} A_{i}(\mathbf{u}) \mathrm{d} \mathbf{u}=\int_{u^{-}}^{u^{+}} K_{n}(\mathbf{u}) \mathrm{d} \mathbf{u}
\end{array}
$$


where $n$ is a coordinate in the direction of the unit normal vector $\mathbf{n}=\left(n_{1}, n_{2}, n_{3}\right)$ on $\gamma_{h}$ and is not uniquely defined yet as it may point to either side of $\gamma_{h}$. Matrix $K_{n}$ is defined as:

$$
K_{n}(\mathbf{u})=\sum_{i=1}^{d} n_{i} A_{i}(\mathbf{u})
$$

For non-conservative systems $\Delta_{n}$ depends on the path chosen (in the $\mathbf{u}$-space) for $\mathbf{u}: \mathbf{u}^{-} \rightarrow \mathbf{u}^{+}$. As the solution is assumed to be smooth, and thus the jump $[u] \rightarrow 0$ for $h \rightarrow 0$, such integral can be approximated as:

$$
\Delta_{n}=H_{n}\left(\mathbf{u}^{-}, \mathbf{u}^{+}\right)[u]+\mathcal{O}\left([u]_{j}^{2}\right),
$$

where $H_{n}$ can be called flux and $H_{n}(\mathbf{a}, \mathbf{a})=K_{n}(\mathbf{a})$. The most obvious choice is:

$$
H_{n}(\mathbf{a}, \mathbf{b})=K_{n}\left(\frac{1}{2}(\mathbf{a}+\mathbf{b})\right),
$$

but others are possible. The same form is obtained by choosing the following linear path:

$$
\mathbf{u}=[\mathbf{u}] \tau+\frac{1}{2}\left(\mathbf{u}^{-}+\mathbf{u}^{+}\right), \quad \tau \in\left[-\frac{1}{2}, \frac{1}{2}\right]
$$

so that:

$$
H_{n}\left(\mathbf{u}^{-}, \mathbf{u}^{+}\right)=\int_{-\frac{1}{2}}^{\frac{1}{2}} K_{n}\left([\mathbf{u}] \tau+\frac{1}{2}\left(\mathbf{u}^{-}+\mathbf{u}^{+}\right) \mathrm{d} \tau,\right.
$$

which leads again to Eq. (43) when the integral is approximated with the mid-point rule. The method therefore takes the form:

$$
\sum_{e=1}^{n_{e}}\left(\int_{\Omega_{e}} \mathbf{v}_{h}^{T}\left(\frac{\partial \mathbf{u}_{h}}{\partial t}+\sum_{i=1}^{d} A_{i}\left(\mathbf{u}_{h}\right) \frac{\partial \mathbf{u}_{h}}{\partial x_{i}}-\mathbf{s}\left(\mathbf{u}_{h}\right)\right) \mathrm{d} \Omega\right)+\int_{\gamma_{h}} \mathbf{v}_{h}^{T} H_{n}\left(\mathbf{u}^{-}, \mathbf{u}^{+}\right)[\mathbf{u}] \mathrm{d} \gamma=0 .
$$

A definition of $\mathbf{v}_{h}$ on $\gamma_{h}$ is still required. The choice is based on the characteristic decomposition of $H_{n}\left(\mathbf{u}^{-}, \mathbf{u}^{+}\right)$.

\section{Characteristic Decomposition}

Characteristic decomposition is based on the solution of the eigenvalue problem for the non-defective matrix $H_{n}\left(\mathbf{u}^{-}, \mathbf{u}^{+}\right)$defined in Eq. (43). The following notation is used:

$$
\begin{array}{ll}
\lambda_{i}: & \text { eigenvalues of } H_{n}\left(\mathbf{u}^{-}, \mathbf{u}^{+}\right), \\
\mathbf{r}_{i}: & \text { right eigenvectors of } H_{n}\left(\mathbf{u}^{-}, \mathbf{u}^{+}\right), \\
\mathbf{l}_{i}: & \text { left eigenvectors of } H_{n}\left(\mathbf{u}^{-}, \mathbf{u}^{+}\right) .
\end{array}
$$

The eigenvectors $\mathbf{r}_{i}$ and $\mathbf{l}_{i}$ can be chosen such that: ${ }^{58}$

$$
\mathbf{l}_{i}^{T} \mathbf{r}_{j}=\delta_{i j}, \quad i, j=1, \ldots, m,
$$

where $\delta_{i j}$ is the Kronecker delta defines as:

$$
\delta_{i j}= \begin{cases}1 & \text { if } i=j \\ 0 & \text { if } i \neq j\end{cases}
$$

The characteristic decomposition of a vector $\mathbf{a}$ is therefore defined with respect to the right and left eigenvectors by writing such a vector as a linear combination of the right $\mathbf{r}_{i}$ or left $\mathbf{l}_{i}$ eigenvectors:

$$
\begin{aligned}
\mathbf{a} & =\sum_{i=1}^{m} a_{i}^{(r)} \mathbf{r}_{i}, & \text { with } a_{i}^{(r)} & =\frac{\mathbf{l}_{i}^{T} \mathbf{a}}{\mathbf{l}_{i}^{T} \mathbf{r}_{i}}, \\
\mathbf{a} & =\sum_{i=1}^{m} a_{i}^{(l)} \mathbf{l}_{i}, & \text { with } a_{i}^{(l)} & =\frac{\mathbf{a}^{T} \mathbf{r}_{i}}{\mathbf{l}_{i}^{T} \mathbf{r}_{i}} .
\end{aligned}
$$


For non-conservative systems, the jump $[\mathbf{u}]$ is decomposed into characteristic components with respect to the $\mathbf{r}_{i}$ base:

$$
[\mathbf{u}]=\sum_{i=1}^{m} \frac{\mathbf{l}_{i}^{T}[\mathbf{u}]}{\mathbf{l}_{i}^{T} \mathbf{r}_{i}} .
$$

Substitution of Eq. (51) in Eq. (46) and using $H_{n}\left(\mathbf{u}^{-}, \mathbf{u}^{+}\right) \mathbf{r}_{i}=\lambda_{i} \mathbf{r}_{i}$ yields the following form for the integral on the boundary:

$$
\int_{\gamma_{h}} \mathbf{v}_{h}^{T} H_{n}\left(\mathbf{u}^{-}, \mathbf{u}^{+}\right)[\mathbf{u}] \mathrm{d} \gamma=\int_{\gamma_{h}} \mathbf{v}_{h}^{T} \sum_{i=1}^{m} \frac{\mathbf{l}_{i}^{T}[\mathbf{u}]}{\mathbf{l}_{i}^{T} \mathbf{r}_{i}} \lambda_{i} \mathbf{r}_{i} \mathrm{~d} \gamma=\int_{\gamma_{h}} \sum_{i=1}^{m} v_{i h}^{(l)} \mathbf{l}_{i}^{T}[\mathbf{u}] \lambda_{i} \mathrm{~d} \gamma,
$$

where $v_{i h}^{(l)}$ is the characteristic component of $\mathbf{v}_{h}$ with respect to the $\mathbf{l}_{i}$ base. Its value is taken as: ${ }^{57}$

$$
v_{i h}^{(l)}= \begin{cases}\alpha v_{i h}^{(l)-}+(1-\alpha) v_{i h}^{(l)+}, & \text { if } \lambda_{i}<0, \\ \alpha v_{i h}^{(l)+}+(1-\alpha) v_{i h}^{(l)-}, & \text { if } \lambda_{i}>0 .\end{cases}
$$

Rearranging Eq. (46) with Eqs. $(52,53)$ into a sum over elements and considering each element independently, yields to the final formulation of the method, which states: for each element $e=1, \ldots, n_{e}$, find $\mathbf{v}_{h} \in V_{h}^{(e)}$ such that:

$$
\begin{aligned}
& \int_{\Omega_{e}} \mathbf{v}_{h}^{T}\left(\frac{\partial \mathbf{u}_{h}}{\partial t}+\sum_{i=1}^{d} A_{i}\left(\mathbf{u}_{h}\right) \frac{\partial \mathbf{u}_{h}}{\partial x_{i}}-\mathbf{s}\left(\mathbf{u}_{h}\right)\right) \mathrm{d} \Omega \\
& \quad+\alpha \sum_{\text {(in) }} \int_{\partial \Omega_{e}} \mathbf{v}_{h}^{T} \frac{\mathbf{l}_{i}^{T}[\mathbf{u}]}{\mathbf{l}_{i}^{T} \mathbf{r}_{i}} \lambda_{i} \mathbf{r}_{i} \mathrm{~d} \gamma+(1-\alpha) \sum_{\text {(out) }} \int_{\partial \Omega_{e}} \mathbf{v}_{h}^{T} \frac{\mathbf{l}_{i}^{T}[\mathbf{u}]}{\mathbf{l}_{i}^{T} \mathbf{r}_{i}} \lambda_{i} \mathbf{r}_{i} \mathrm{~d} \gamma=0, \quad \forall \mathbf{v}_{h} \in V_{h}^{(e)},
\end{aligned}
$$

where inner summation is a sum over all $i$ with $\lambda_{i}<0$, outer summation is a sum over all $i$ with $\lambda_{i}>0$ and $\mathbf{n}$ unit normal vector used to define $K_{n}$ is pointing out from the volume $\Omega_{e}$.

\section{Discretization and Solution of the System}

The approximate solution $\mathbf{u}_{h}$ and the test function $\mathbf{v}_{h}$ are expanded formally in terms of the basis function as: ${ }^{59}$

$$
\mathbf{u}_{h}(\mathbf{x}, t)=\mathbf{u}_{j k}(t) b_{k}(\mathbf{x}), \quad \mathbf{v}_{h}(\mathbf{x}, t)=b_{m}(\mathbf{x}),
$$

where Einstein summation convention is used. Here $b$ are the linearly independent basis functions (i.e. monomials), $k$ and $m$ index denote the basis function considered $k, m=1,2, \ldots, N(p, d)$, while $j$ index denotes the $j$-th element $j=1,2, \ldots N_{e}$, with $N_{e}$ number of elements.

The reader is referred to previous studies ${ }^{53,20}$ for the detailed derivation of the final discrete equation that reads as follows:

$$
\frac{\mathrm{d} \mathbf{u}_{j k}}{\mathrm{~d} t}+\frac{1}{J_{j}}\left[M_{m k}^{-1} G_{k m l} A_{j l} \mathbf{u}_{j k}+\alpha \sum_{(\text {in }) \epsilon} M_{m k}^{-1} \tilde{B}_{\epsilon} H_{j} \mathbf{u}_{j k}+(1-\alpha) \sum_{(\text {out }) \epsilon} M_{m k}^{-1} \tilde{B}_{\epsilon} H_{j} \mathbf{u}_{j k}\right]=\mathbf{s}_{j k},
$$

with $J_{j}$ is the Jacobian matrix, and $\alpha$ is the weight factor the pre-computed and stored matrices given below

$$
M_{m k} \equiv \int_{\hat{\Omega}} b_{k}(\boldsymbol{\xi}) b_{m}(\boldsymbol{\xi}) d \hat{\Omega}, \quad G_{m k l} \equiv \int_{\hat{\Omega}} \frac{\partial b_{m}(\boldsymbol{\xi})}{\partial \xi_{l}} b_{k} d \hat{\Omega}, \quad \tilde{B}_{\epsilon} \equiv \int_{\Gamma_{\epsilon}} b_{m} \bar{b}_{k} d \bar{\Gamma}
$$

The explicit four stage Runge-Kutta Scheme is used for time discretization. ${ }^{53,60}$

$$
\begin{gathered}
\mathbf{u}_{j}^{n, 0}=\mathbf{u}_{j}^{n}, \\
\mathbf{u}_{j}^{n, k}=u_{j}^{n}+\gamma_{k} \Delta t K \mathbf{u}_{j}^{n, k-1}, \quad k=1, . . N, \\
\mathbf{u}_{j}^{n+1}=\mathbf{u}_{j}^{N, n},
\end{gathered}
$$

where $N=4$, and $\gamma_{1}=1 / 4, \gamma_{2}=1 / 3, \gamma_{3}=1 / 2, \gamma_{4}=1$. 


\section{Results}

In this section, the numerical solutions of the individual components namely viscous flow solution and potential flow solution are verified. Later on, the solution of the coupled system is demonstrated on chosen test cases.

\section{III.A. Viscous flow solution}

In previous studies the DG method applied to various academic test cases and the convergence rate of both the space and time discretization are presented (up to $4 t h$ order of accuracy) and the method is verified (see i.e. ${ }^{61,19,20}$ ). Within this study the focus is mainly on applying the numerical method on the IBL equations. To this end various test cases on flat plate and various airfoils (and angles of attacks) are shown in the following sections. Converged steady results are compared with the aforementioned solutions and the accuracy of the method is demonstrated. It is important to note that although the numerical method is developed for an arbitrary order of accuracy it is a tedious work to discretize the equation set used to close the system for higher order of accuracies and therefore their accuracy is kept at second order which is bounding for the whole system. It should also be noted that since the aim of this section is to demonstrate the solutions obtained by the viscous flow solver only, the edge velocity distribution is assumed to be prescribed (only in this section) and furthermore these velocity distributions are either set to certain (analytical) values or obtained from other numerical solutions (i.e. XFOIL) when necessary.

\section{III.A.1. Laminar flow over a flat plate}

An interesting preliminary test case for the modeling of a boundary layer flow with the integral boundary layer equations is a laminar flow over a flat plate. The reason for this is that an analytical solution is available for this type of flow and a proper comparison and analysis of the discretisation method can be performed. The exact solution of the flat plate is obtained by solving the Blasius equation (e.g. White ${ }^{62}$ ). The introduction of closure relations, Eqs. (17,18 and 19), which are optimized for airfoils, introduces an error compared to the exact solution. For this test case a reference solution is derived in $^{36}$ and here recalled together with the exact solution. The edge velocity prescribed for a flat plate is $u_{e}=V_{\infty}$, where $V_{\infty}$ is the

\begin{tabular}{lll}
\hline Variable & Blasius & IBL \\
\hline$\delta^{*}$ & $1.72079 \sqrt{\frac{x L}{R e_{\infty}}}$ & $1.71029 \sqrt{\frac{x L}{R e_{\infty}}}$ \\
$\theta$ & $0.66411 \sqrt{\frac{x L}{R e_{\infty}}}$ & $0.66599 \sqrt{\frac{x L}{R e_{\infty}}}$ \\
$H$ & 2.59110 & 2.56805
\end{tabular}

Table 1. Solutions for a flat plate boundary layer Flow. ${ }^{36}$

\begin{tabular}{l|c|c}
\hline & $p=0$ & $p=1$ \\
\hline$N=100$ & - & - \\
$N=200$ & 1.0067 & 1.9870 \\
$N=400$ & 1.0021 & 1.9821 \\
\hline Expected & 1.00 & 2.00
\end{tabular}

Table 2. Order of Accuracy of DG discretization for boundary layer flow over a flat plate.

free stream velocity. The unsteady simulation is hence performed until a steady solution is obtained for a Reynolds number of $R e=10^{5}$. The effectiveness of the method can be perceived by a simulation with very few elements. In Fig. (3(a)), the solution converged to a steady state is obtained at the end of the simulation with $N=10$ elements for the basis functions of degree $p=0$ and $p=1$ is shown, together with the analytical solution.

As can be seen from Fig. (3(a)), already a very coarse mesh give results very close to the exact solution. The first element is of course the most problematic and where the numerical solution is less accurate due to the infinite slope which can not be well represented by low order polynomials. The problem is reduced by $p$-refinement or $h$-refinement. For $p=0$ the method actually reduces to a finite volume scheme, but an increase on the degree of the basis function increases the accuracy of the solution faster than a mesh refinement.

As previously discussed, due to the complex form and not exact derivation of the closure relations, the practical implementation of the method is currently limited up to second order accuracy. The order of accuracy is tested with a grid refinement study. The convergence study is accomplished by evaluating the solution on an interrogation mesh with 10000 common points used for the meshes under analysis, which have 100, 200 and 400 elements in the streamwise $\mathrm{x}$-direction. The number of evaluation points per element is 


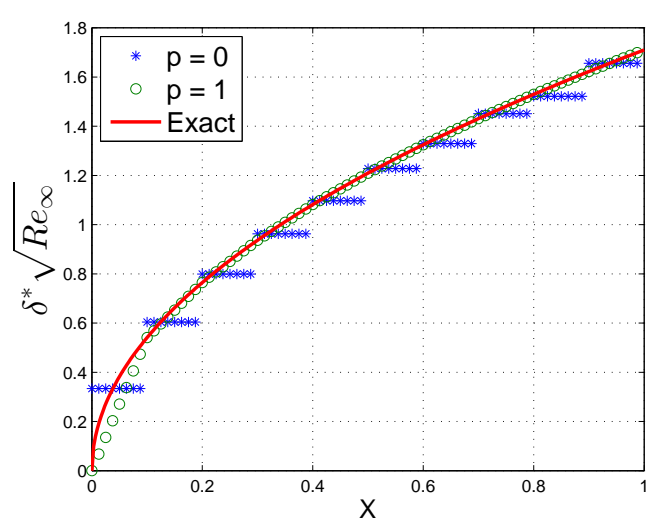

(a) $\mathrm{N}=10$ elements.

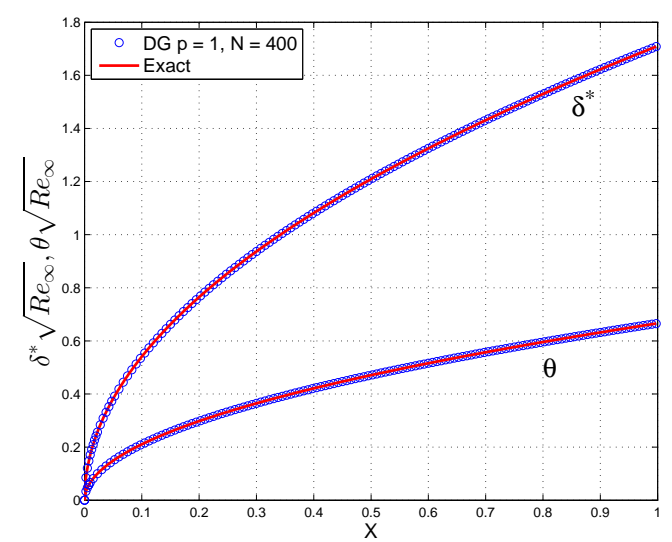

(b) $\mathrm{N}=400$ elements.

Figure 3. Comparison of the numerical solution with the analytical solution for the flow over a flat plate for the displacement thickness, $\delta^{*}$, and for the momentum thickness, $\theta$.

therefore 100,50 , and 25 respectively. The $L_{2}$-norm is employed to measure the error $e(x)=\delta^{*}(x)-\delta_{\text {exact }}^{*}(x)$ :

$$
\|e(x)\|_{L_{2}}=\left\{\frac{1}{L} \int_{0}^{L}\left[\delta^{*}(x)-\delta_{\text {exact }}^{*}(x)\right]^{2} d x\right\}^{1 / 2}=\left\{\frac{1}{10000} \sum_{j=1}^{10000}\left[\delta^{*}\left(x_{j}\right)-\delta_{\text {exact }}^{*}\left(x_{j}\right)\right]^{2} d x\right\}^{1 / 2} .
$$

Fig. (4) shows the $L_{2}$-norm of the error for the variable $\delta^{*}$ (similar results are obtained for the other variable $\theta$ ). Lesaint and Raviart ${ }^{63}$ demonstrated that the method can be $h^{p+1}$ accurate for cartesian grids, where $h$ is a reference length for the elements size. Computation of the slopes of the error norm in a logarithmic graph gives exactly the accuracy expected as shown in table (III.A.1).

In Fig. (3(b)) finally the displacement thickness $\delta^{*}$ and momentum thickness $\theta$ for $p=1$ and the finest grid with $N=400$ elements are shown. The results agree very well with the analytical solution.

\section{III.A.2. Turbulent flow over a flat plate}

As a second test case turbulent flow over a flat plate is considered. The experimental data used to validate the numerical solution are from Wieghardt and Tillmann ${ }^{64}$ as identified with the flow number 1400 . Free-stream velocity is $u_{e}=33 \mathrm{~m} / \mathrm{s}$ with no pressure gradient along the five meter long flat plate. The corresponding Reynolds number is $R e_{L}=1.0927 \times 10^{7}$. For the unsteady simulations the simulation time is chosen to be long enough such that the boundary layer may be considered to converge to a steady state. In Fig. (5) comparisons of the nu-

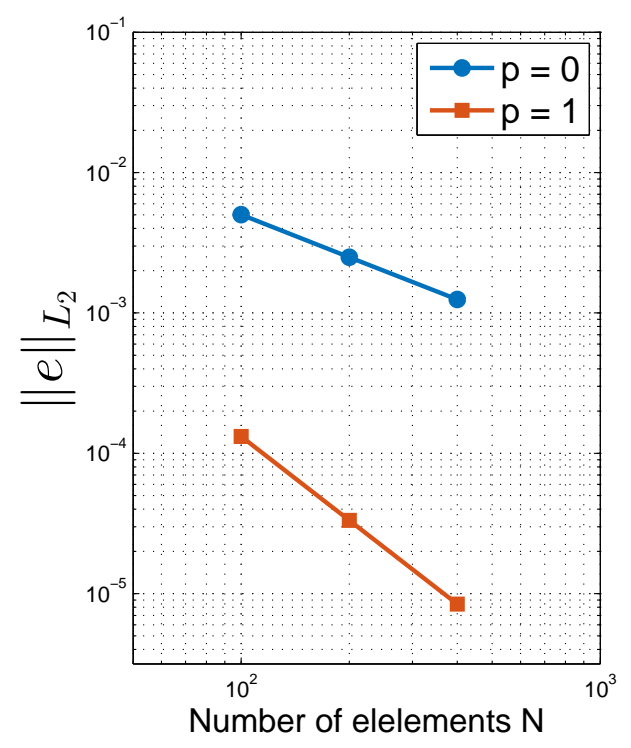

Figure 4. $L_{2}$ error norms for the steady state solution of the flat plate test case for different degree $p$ of the basis functions. coefficients $C_{f}$ to the experimental data are shown. Numerical solution is in a very good agreement with the experimental data for the turbulent flow over a flat plate case.

\section{III.A.3. Laminar flow over airfoil profiles}

Unsteady simulation of a flow over a NACA 0009 airfoil profile is presented. The airfoil is considered to set into motion instantaneously where the boundary layer is developed in time. A steady solution is obtained with the aerodynamic design software XFOIL ${ }^{11}$ in order to compare the results with the ones obtained by 


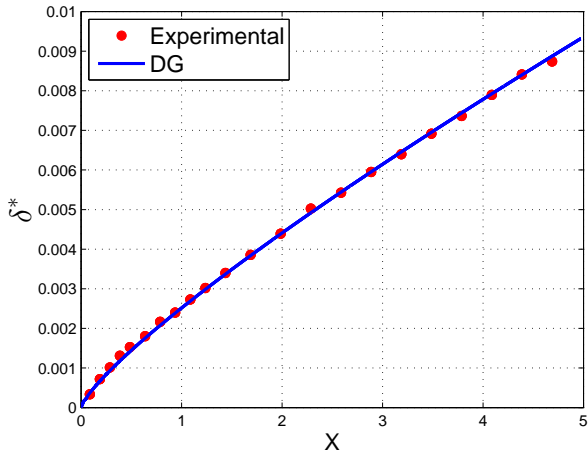

(a) Displacement Thickness $\delta^{*}$.

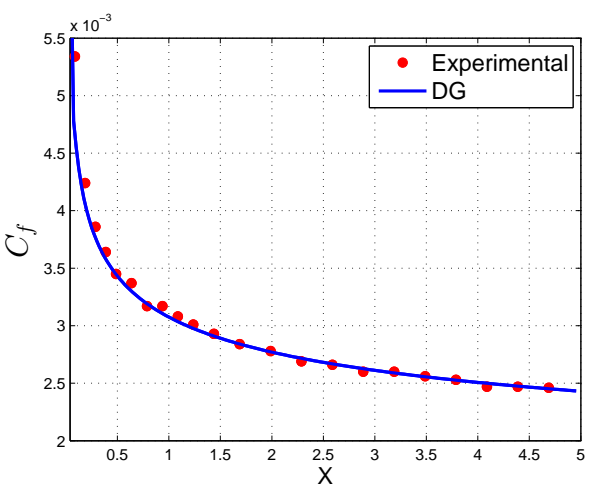

(b) Friction Coefficient $C_{f}$.

Figure 5. Turbulent boundary layer over a flat plate with $R e=1.09 \times 10^{7}$, denoted as Flow $1400 .^{64}$

the present method. NACA 0009 is a symmetric airfoil with maximum thickness of 9 percent of the chord. Reynolds number for the numerical simulation is kept relatively low $\left(R e=10^{4}\right)$ in order to ensure the flow to be fully laminar. A simulation with zero angle-of-attack is performed. Since the airfoil geometry is symmetric only one side of the solution is presented. In Fig. (6) the evaluation of the solution for $\delta^{*}$ in time is presented. Since the initial and boundary conditions are not time dependent it is expected that the solution converges to a steady state solution as can be seen from the comparison with the solution obtained by XFOIL.

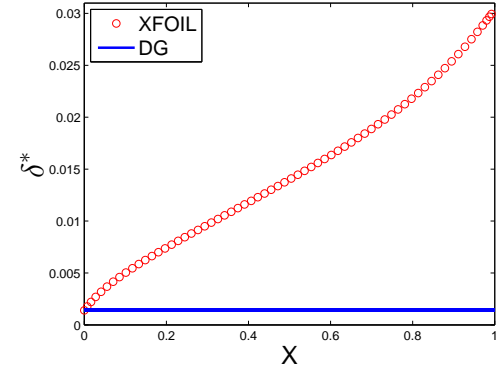

(a) Initial condition.

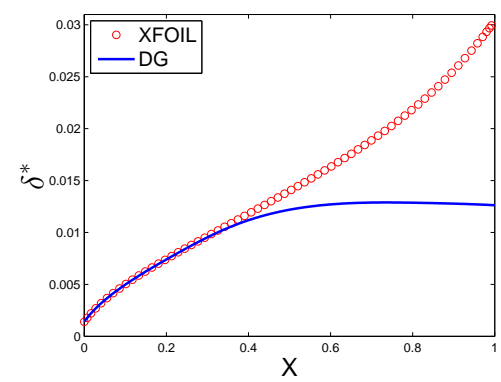

(c) $t=2.0 \mathrm{~s}$

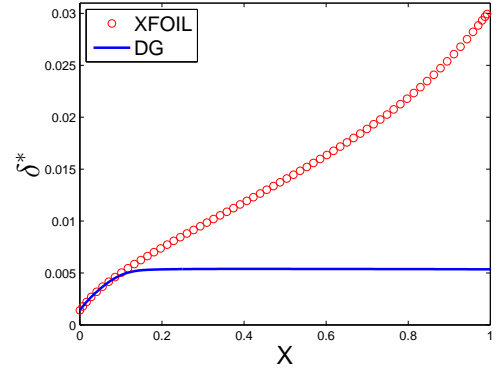

(b) $t=0.2 \mathrm{~s}$

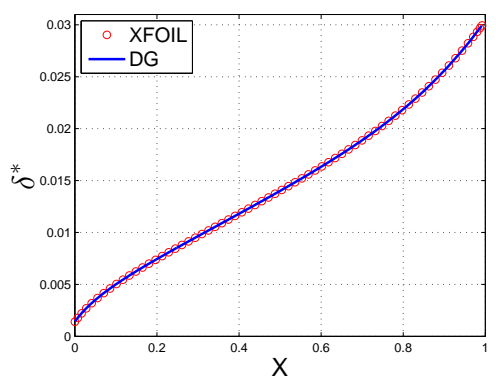

(d) Steady solution

Figure 6. Unsteady simulation for NACA 0009 at $R e=10^{4}$ and $\alpha=0^{\circ}$. Time evaluation of the displacement thickness $\delta^{*}$ is compared to the results obtained by XFOIL.

In order to have some asymmetries on suction and pressure side, a cambered airfoil NACA 2205 is then tested for 0 deg angle of attack and for the Reynolds number of $10^{4}$.

Distributions of displacement and momentum thickness along the cord for the solution converged to a steady state are shown in Fig. (7) together with XFOIL results which shows a good agreement. 


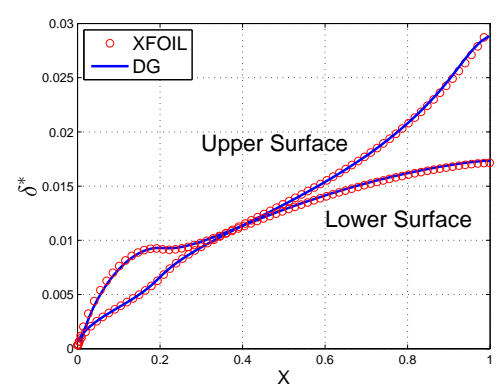

(a) Displacement Thickness $\delta^{*}$.

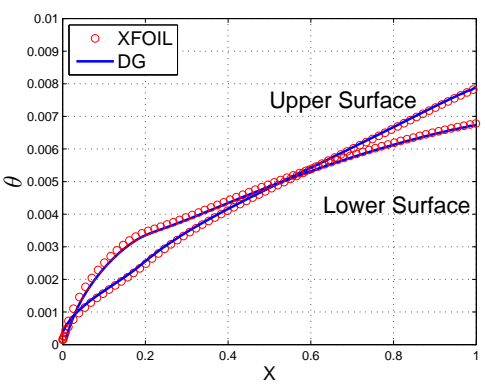

(b) Momentum Thickness $\theta$.

Figure 7. A comparison of the results converged to steady state for NACA 2205 airfoil profile for $R e=10^{4}$ and $\alpha=0^{\circ}$ obtained by the unsteady simulation with the results obtained by XFOIL.

\section{III.B. Potential flow solution}

The panel method based on the linear-strength vorticity singularity is tested on the $12 \%$ thick van de Vooren airfoil (Fig. (9)). Fig. (8(a)) and Fig. (8(b)) show the comparison of the numerical solutions for the pressure coefficients obtained by the panel method with the one obtained by XFOIL at angles of attack 1 and 5 degree respectively. It can be clearly seen that the numerical solution is in very good agreement with XFOIL. Fig. (9)

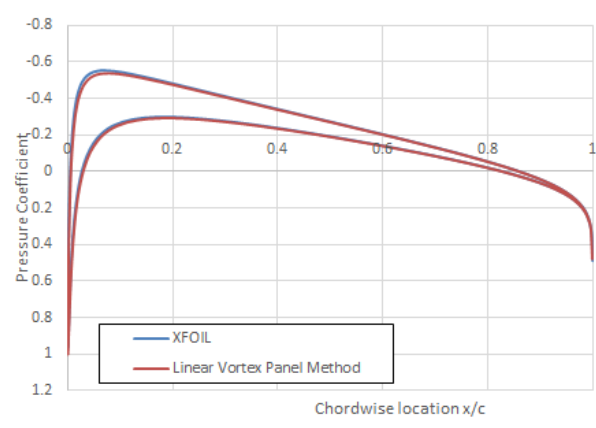

(a) Angle of Attack $=1^{\circ}$.

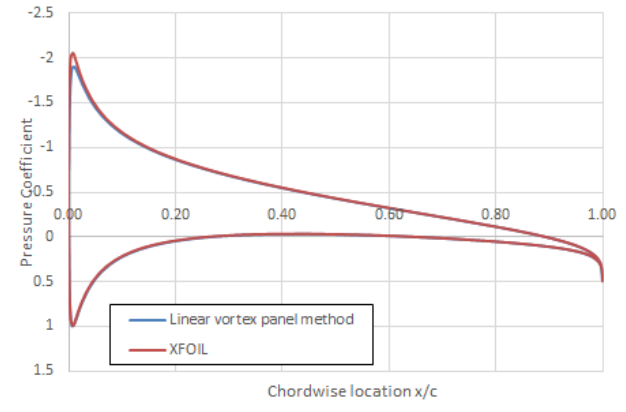

(b) Angle of Attack $=5^{\circ}$.

Figure 8. Comparison of the pressure coefficient between Panel method and XFOIL.

shows the wake trajectories that are computed for different angles of attacks.

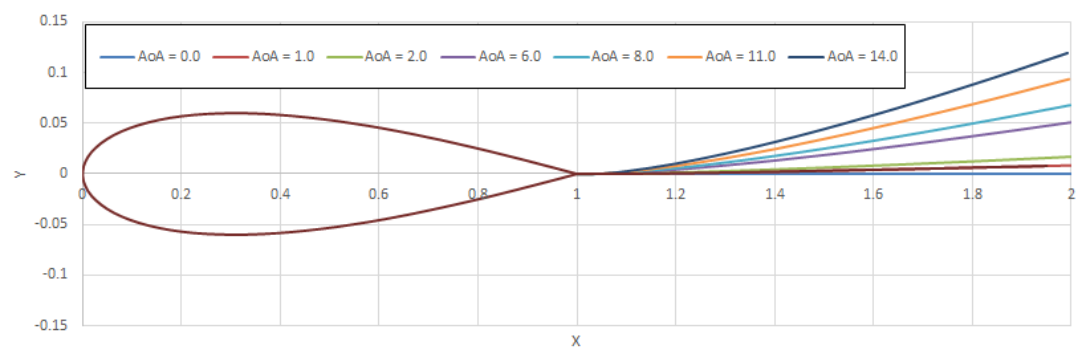

Figure 9. Airfoil with wake trajectory for various angles of attack.

\section{III.C. Solution with the coupled system}

The individual numerical methods presented above coupled with the unsteady form of the quasi-simultaneous viscous-inviscid interaction scheme to form the complete interacting boundary layer method. The method is applied to a flow over a NACA 63418 airfoil profile for the cases where a natural transition to turbulent flow is allowed and also where the flow is triggered at the leading edge to become fully turbulent. The 
numerical solutions are obtained for the Reynolds number of $3.0 \times 10^{6}$ and for the angles of attack of $-1^{\circ}$, $1^{\circ}$ and $3^{\circ}$ where mild separation is expected. Fig. (10(a)) shows a comparison of the solution for the lift coefficient to the experimental data and the numerical solutions obtained by other numerical methods. A similar comparison is made for the drag coefficient in Fig. (10(b)). Although for the present method only three representative angles of attacks are chosen it can be seen that for the linear part of both polar curves lift coefficient is slightly over-predicted while the drag coefficient is slightly under-predicted. It should be noted that the closure equation set used in the aerodynamic design tools (like XFOIL) are usually fine tuned by considering many test cases which is not (yet) the case for the presented method.

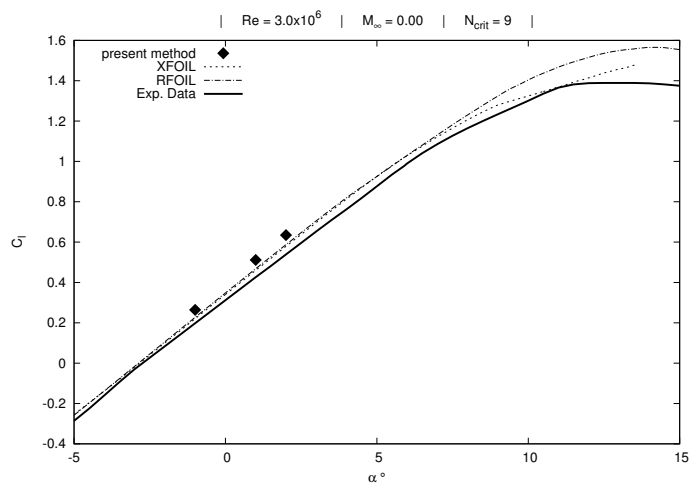

(a) Lift.

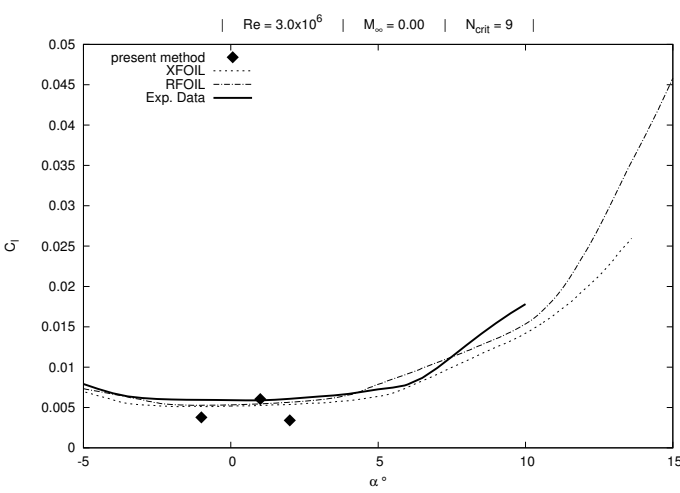

(b) Drag.

Figure 10. Comparison of numerical solution to the experimental data and other numerical solutions for lift and drag.

In Fig. (11(a)) a comparison of the numerical solution for the displacement thickness, $\delta^{*}$ is shown to the numerical solution obtained by XFOIL where the flow naturally transitions from laminar to turbulent conditions for NACA 63418 airfoil for $R e=3.0 \times 10^{6}$ and $\alpha=1^{\circ}$. The present (unsteady) method is simulated for a long enough time to achieve a steady state solution. It is interesting to note that the wake simulation in the present method is performed in a rather unconventional manner where two layer of panels are introduced (i.e. can be interpreted as pressure and the suction sides of the wake). This implementation can be seen in Fig. (11(a)) clearly. The upper (suction side) end location of the wake is located to $x=-1$ and continues until $x=0$ where the trailing edge of the suction side starts. The leading edge of the airfoil is around $x=1$ and the pressure side of the airfoil geometry continues until around $x=2$ reaching the trailing edge of the body on the pressure side. Finally the lower side of the wake (pressure side) extends until $x=3$. Within this setting when the upper and lower sides of the wake is examined a difference in the solution (displacement thickness) can be noticed. This result is expected for non-symmetric airfoils. Furthermore from the comparison it can be seen that the transition onset and the behavior of the transition is different for both solutions. Although the transition model applied involves unsteady terms this model needs a further attention. In order to avoid the use of transition model the same test case is considered for a fully turbulent flow condition where the solution is compared to the XFOIL result as presented in Fig. (11(b)). Considering the differences between the present method and XFOIL one naturally does not expect to see an exact match in the solutions. It can be concluded that the solution obtained with the present method is in good agreement with XFOIL.

\section{Conclusion}

Within this study an unsteady, two-dimensional interacting boundary layer method is presented for the incompressible flow around wind turbine rotor blade sections. The main approach is to divide the flow field in to two regions; the one in the vicinity of the surface where the viscosity is effective (so called boundary layer) and the one away from the surface where the flow can be assumed as inviscid. The solutions obtained from these two regions are matched with a quasi-simultaneous viscous-inviscid interaction scheme.

The unsteady integral boundary layer equations are presented together with the laminar and turbulent closure sets. For the turbulent flow conditions, an unsteady shear lag equation is employed. Laminar to turbulent transition is modeled with the $e^{N}$ method where an unsteady term is introduced to the amplification rate $(N)$. The potential flow is solved by using the linear-strength vortex panel method. 


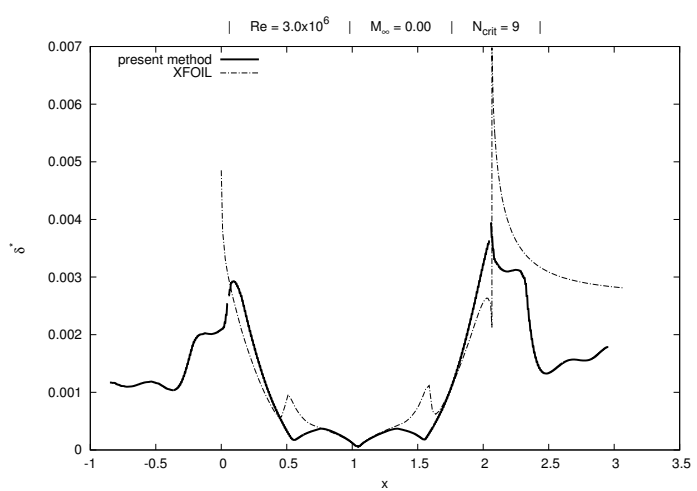

(a) Natural transition.

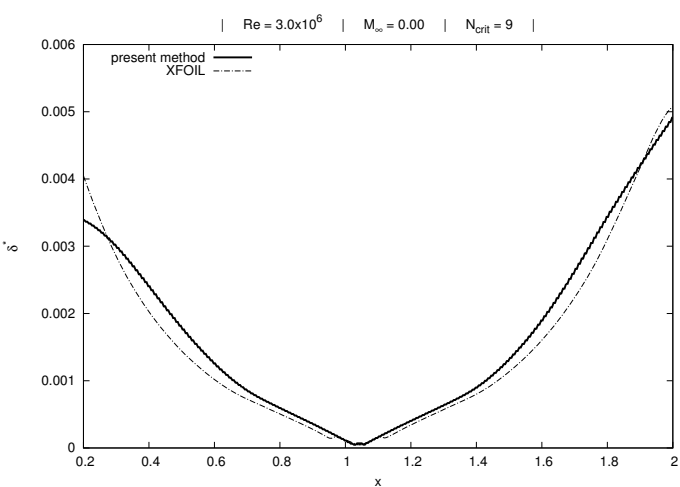

(b) Fully turbulent.

Figure 11. Comparison of the solution for the displacement thickness, $\delta^{*}$ to the solution obtained by XFOIL for natural transition and fully turbulent flow conditions for NACA 63418 airfoil for $R e=3.0 \times 10^{6}$ and $\alpha=1^{\circ}$.

It is shown that introducing the interaction scheme leads to non-conservative mechanisms in the system that requires a special treatment for these non-conservative flux terms. The viscous boundary layer flow represented by the unsteady integral boundary layer equations is solved using a high-order quadrature-free discontinuous Galerkin method, where the method is extended to handle aforementioned non-conservative flux terms. Furthermore it is shown that this numerical method achieves the designed order of accuracy for smooth problems.

The individual numerical solution methods are verified on various test cases including laminar and turbulent flow over a flat plate and airfoil profiles. The time dependent solution of the developing boundary layer is presented. Later, the coupled system is applied on a test case and the results are compared to experimental data and the numerical results obtained from other numerical solution methods. It is shown that the developed numerical method is in good agreement with experimental data and results from other numerical methods.

\section{References}

${ }^{1}$ Hoeijmakers, H. W. M., "Panel Methods for Aerodynamic Analysis and Design," Special Course on Engineering Methods in Aerodynamic Analysis and Design of Aircraft, No. AGARD Report R-783, 1991.

${ }^{2}$ van Garrel, A., "Development of a Wind Turbine Rotor Flow Panel Method," Report ECN-E-11-071, ECN, january 2012.

${ }^{3}$ van Garrel, A., "Development of a wind turbine aerodynamics simulation module," ECN rapport ECN-C-03-079, ECN, August 2003.

${ }^{4}$ Cebeci, T. and Cousteix, J., Modeling and computation of boundary-layer flows, Springer, Berlin Heidelberg, 2nd ed., 2005, ISBN-3-540-65010-5.

${ }^{5}$ Goldstein, S., "On laminar boundary-layer flow near a position of separation," Quarterly Journal of Mechanics and Applied Mathematics, Vol. 1, No. 1, 1948, pp. 43-69.

${ }^{6}$ van Dommelen, L. and Shen, S., "The spontaneous generation of the singularity in a separating laminar boundary layer," Journal of Computational Physics, Vol. 38, No. 2, 1980, pp. 125 - 140.

${ }^{7}$ Matsushita, M. and Akamatsu, T., "Numerical computation of unsteady laminar boundary layers with separation using one-parameter integral method," JSME, Vol. 28, No. 240, June 1985, pp. 1044-1048.

${ }^{8}$ Coenen, E. G. M., Viscous-Inviscid Interaction with the Quasi-Simultaneous Method for $2 D$ and $3 D$ Aerodynamic Flow, Ph.D. thesis, Rijksuniversiteit Groningen, 2001, ISBN: 90-367-1472-9.

${ }^{9}$ Veldman, A. E. P., "New, Quasi-Simultaneous Method to Calculate Interacting Boundary Layers," AIA A journal, Vol. 19, No. 1, 1981, pp. 79-85.

${ }^{10}$ Drela, M., Two-Dimensional Transonic Aerodynamic Design and Analysis using the Euler Equations, Ph.D. thesis, Massachusetts Institute of Technology, 1984.

${ }^{11}$ Drela, M., "XFOIL: An analysis and design system for low Reynolds number airfoils," 1989.

${ }^{12}$ Rooij, R. v., "Modification of the boundary layer in XFOIL for improved stall prediction," Report IW-96087R, Delft University of Technology, Delft, The Netherlands, September 1996.

${ }^{13}$ Snel, H., Houwink, R., and Bosschers, J., "Sectional prediction of lift coefficients on rotating wind turbine blades in stall," Tech. Rep. ECN-C-93-052, ECN, 1993.

${ }^{14}$ Nishida, B., Fuly simultaneous coupling of the full potential equation and the integral boundary layer equations in three dimensions, Ph.D. thesis, Massachusetts Institute of Technology, February 1996. 
${ }^{15}$ Drela, M., "Three-Dimensional Integral Boundary Layer Formulation for General Configurations," Fluid Dynamics and Co-located Conferences, American Institute of Aeronautics and Astronautics, June 2013.

$\checkmark{ }^{16}$ Cebeci, T., Platzer, M. F., Jang, H. M., and Chen, H. H., "Inviscid-viscous interaction approach to the calculation of dynamic stall initiation on airfoils," Journal of Turbomachinery, Vol. 115, No. 4, 1993, pp. 714-723.

${ }^{17}$ Zhang, Z., Liu, F., and Schuster, D., "Calculations of Unsteady Flow and Flutter by an Euler and Integral Boundary-Layer Method on Cartesian Grids," Proc. 22nd Applied Aerodynamics Conference, AIAA, Aug. 2004.

${ }^{18}$ Garcia, N. R., Unsteady Viscous-Inviscid Interaction Technique for Wind Turbine Airfo, Ph.D. thesis, DTU, 2011.

${ }^{19}$ Seubers, J. H., Path-consisten Schemes for Interacting Boundary Layers, Master's thesis, Delft University of Technology, The Netherlands, 2014

${ }^{20}$ Passalacqua, F., Implementation of unsteady two-dimensional interacting boundary layer method, Master's thesis, Politecnico di Milano, 2015.

${ }^{21}$ von Kármán, T., "On laminar and turbulent friction," Tech. Rep. TM-1092, NACA, 1946.

${ }^{22}$ Rosenhead, L., Laminar boundary layers, Dover, New York, 1966.

${ }^{23}$ White, F., Viscous Fluid Flow, McGraw-Hill, 2nd ed., 1991.

- ${ }^{24}$ Swafford, T., "Analytical approximation of two-dimensional separated turbulent boundary layer velocity profiles," $A I A A$ Journal, Vol. 26, 1983, pp. 923-926.

${ }^{25}$ Mugal, B., Integral methods for three-dimensional boundary-layers, Ph.D. thesis, MIT, 1998.

26 Lighthill, M. J., "On Displacement Thickness," Journal of Fluid Mechanics, Vol. 4, No. 04, August 1958, pp. 383-392.

${ }^{27}$ Tetervin, N., "Boundary-layer momentum equations for three-dimensional flow," Tech. rep., 1947.

${ }^{28}$ Matsushita, M., Murata, S., and Akamatsu, T., "Studies on boundary-layer separation in unsteady flows using an integral method," J.Fluid Mech., Vol. 149, 1984, pp. 477-501.

${ }^{29}$ Swafford, T., "Analytical approximation of two-dimensional seperated turbulent boundary-layer velocity profiles," AIAA Journal, Vol. 21, No. 6, 1963, pp. 923-926.

${ }^{30}$ Seubers, J. H., "Project Proposal Modelling of Unsteady Boundary Layers," proposal, 112013.

${ }^{31}$ Goldberg, P., "Upstream history and apparent stress in turbulent boundary layers." Tech. rep., DTIC Document, 1966.

${ }^{32}$ Green, J., Weeks, D., and Brooman, J., "Prediction of turbulent boundary layer and wakes in compressible flow by a lag-entrainment method," R\&M 3791, Aeronautical research council, 1977.

${ }^{33}$ Özdemir, H., "Development of a discontinuous Galerkin method for the unsteady integral boundary layer equations." Tech. rep., Energy Research Centre of the Netherlands, Petten, The Netherlands, 2010.

${ }^{34} \mathrm{Ye}$, B., The Modeling of Laminar-to-Turbulent Transition for Unsteady Integral Boundary Layer Equations with HighOrder Discontinous Galerkin Method, Master's thesis, Delft University of Technology, 2015.

- ${ }^{35}$ Thomas, J., "Integral Boundary-Layer Models for Turbulent Separated Flows," AIAA 14th Fluid and Plasma Dynamics Conference, Snwomass, CO, U.S.A., June 1984.

${ }^{36}$ van den Boogard, E., High-Order Discontinuos Galerkin Method for Unstready Integral Boundary Layer Equation, Ph.D. thesis, Delft University of Technology, 2010.

${ }^{37}$ Ingen, J. V., "Theoretical and Experimental investigations of incompressible laminar boundary layers with and without suction." Tech. rep., 1965.

${ }^{38}$ Smith, A. and Gamberoni, N., "Transition, pressure gradient and stability theory." Tech. rep., Douglas Aircraft Company, El Segundo Division, 1956.

${ }^{39}$ Schlichting, H. and Gersten, K., Boundary Layer Theory, Springer, eigth ed., 2000.

${ }^{40}$ Drela, M., "MISES Implementation of modified Abu-Ghanam/Shaw transition criterion," Report, MIT Aero-Astro, 1995.

${ }^{41}$ Ingen, J. V., "A new $e^{N}$ method for transition prediction. Historical review of work at TU Delft." AIAA Journal, , No. 3830:2008, 2008.

${ }^{42}$ Drela, M. and Giles, B., "Viscous-Inviscid Analysis of Transonic and Low Reynolds Number Airfoils," AIAA Journal, Vol. 25, No. 10, 1987, pp. 1347-1355.

${ }^{43}$ Katz, J. and Plotkin, A., Low-Speed Aerodynamics, Cambridge Aerospace Series, Cambridge University Press, 2001.

${ }^{44}$ Koodly-Ravishankara, A., Implementation of two dimensional unsteady interacting boundary layer method, Master's thesis, Purdue University, to be published.

${ }^{45}$ Haciahmetoglu, M., Investigation of unsteady viscous-inviscid interaction (draft), Master's thesis, Delft University of Technology, Kluyverweg 1, April 2013.

${ }^{46}$ Veldman, A. E. P., "Boundary Layers in FLuids," Lecture Notes in Applied Mathematics.

${ }^{47}$ Brune, G., Rubbert, P., and Nark, Junior, T. C., "A New Approach to Inviscid Flow / Boundary Layer Matching," 7th Fluid and Plasma Dynamics Conference, AIAA, Palo Alto, CA, U.S.A., 1974.

${ }^{48}$ Williams, B. R. and Smith, P. D., "Coupling Procedures for Viscous-Inviscid Interaction for Attached and Separated Flows on Swept and Tapered Wings," Numerical and Physical Aspects of Aerodynamic Flows IV, edited by T. Cebeci, Springer Berlin Heidelberg, 1990, pp. 53-70.

${ }^{49}$ Veldman, A. E. P., "A simple interaction law for viscousinviscid interaction," Journal of Engineering Mathematics, Vol. 65, No. 4, 2009, pp. 367-383.

${ }^{50}$ Basu, B. C. and Hancock, G. J., "The Unsteady Motion of a two-dimensional Aerofoil in Incompressible Inviscid Flow," Journal of Fluid Mechanics, Vol. 87, No. 01, 1978, pp. 159-178.

${ }^{51}$ Atkins, H. L. and Shu, C.-W., "Quadrature-Free Implementation of the Discontinuos Galerkin Method for Hyperbolic Equations," AIAA-Paper 96-1683, 1996.

52 Cockburn, B. and Shu, C.-W., "Runge-Kutta Discontinuous Galerkin Methods for Convection-Dominated Problems," Journal of Scientific Computing, Vol. 16, No. 3, 2001, pp. 173-261.

${ }^{53}$ Özdemir, H., High-order Discontinuous Galerkin Method on Hexahedral elements for aeroacoustics, Ph.D. thesis, University of Twente, 2006. 
${ }^{54}$ Özdemir, H. and van den Boogaard, E., "Solving the integral boundary layer equations with discontinuous Galerkin method,", EWEA, Brussels, Belgium, 2011.

${ }^{55}$ Dal Maso, G., Lefloch, P. G., and Murat, F., "Definition and weak stability of nonconservative products," Journal de mathématiques pures et appliquées, Vol. 74, No. 6, 1995, pp. 483-548.

${ }^{56}$ Whitham, G. B., Linear and Non linear Waves, A Wiley-Interscience Series of Texts, Monographs \& Tracts, John Wiley $\&$ and Sons, 1974.

${ }^{57}$ Hulsen, M. A., "The Discontinuous Galerkin Method with Explicit Runge-Kutta Time Integration for Hyperbolic and Parabolic Systems with Source Terms," Tech. Rep. MEMT 19, Delft University of Technology, Laboratory for Aero and Hydrodynamics, The Netherlands, 1991.

${ }^{58}$ Hirsch, C., Numerical Computation of Internal and External Flows: Computational Methods for Inviscid and Viscous Flows, Wiley series in numerical methods in engineering, John Wiley \& Sons, 1990.

${ }^{59}$ Lowrie, R. B., Roe, P. L., and van Leer, B., "A Space-Time Discontinuos Galerkin Method for the Time-Accurate Numerical SOlution of Hyperbolic Conservation Laws," AIAA-Paper 95-1658, June 1995.

${ }^{60}$ Jameson, A., Schmidt, W., and Turkel, E., "Numerical Solution of the Euler Equations by Finite Volume Methods Using Runge-Kutta Time-Stepping Schemes," AIAA Paper 1981-1259, 1981, p. 1.

${ }^{61}$ Özdemir, H., Hagmeijer, R., and Hoeijmakers, H., "Verification of the higher order Discontinuous Galerkin Method on hexahedral elements," Comptes Rendus Mecanique, Vol. 333, 2005, pp. 719-725.

${ }^{62}$ White, F., Viscous fluid flow, McGraw-Hill, 3rd ed., 2006, ISBN 0-07-124493-X.

${ }^{63}$ Lesaint, P. and Raviart, P., "On a Finite Element Method for Solving the Neutron Transport Equation," Mathematical ASpects of Finite Elements in Partial Differential Equaitons, 1974, pp. 89-145.

${ }^{64}$ Wieghardt, K. and Tillmann, W., On the Turbulent Friction Layer for Rising Pressure, Technical memorandum 1314, National Advisory Committee for Aeronautics, 1953, Translation of ZWB Untersuchungen und Mtteilungen, Nr. 6617, November 20, 1944. 